\title{
Skin dosimetry of patients during interventional cardiology procedures in the Czech Republic
}

\author{
${ }^{1}$ National Radiation Protection Institute, Prague, Czech Republic \\ ${ }^{2}$ Faculty of Nuclear Sciences and Physical Engineering, Prague, Czech Republic \\ e-mail: leos.novak@suro.cz
}

\begin{abstract}
The aim of the study is to determine distribution of air kerma-area product, fluoro time and number of frames values for the two most frequent procedures in the interventional cardiology, to reconstruct skin dose distributions for some patients undergoing coronarography and percutaneous transluminal coronary angioplasty procedures. Patient dose data were obtained from X-ray unit dose monitoring software report from one hospital and the reconstructions were performed in MATLAB. Dependence of maximum skin dose on air kerma-area product, fluoro time and number of frames was determined to assess trigger levels of these quantities, which can indicate possible exceeding of the 2 Gy skin dose threshold.
\end{abstract}

Key words: coronarography, PTCA, kerma-area product, skin dose distribution, maximum skin dose.

\section{Introduction}

The ischaemic heart disease (IHS) is one of the most often cause of deaths in developed countries of the world. The IHS is characterized by reduced blood supply to the heart muscle, usually due to atherosclerosis of the coronary arteries. An investigation of coronary arteries, the coronary angiography (CA), and a treatment of some arteries, the percutaneous transluminal coronary angioplasty (PTCA), are usually performed in a cardiac catheterization laboratory. The CA and the PTCA are the most frequent procedures in interventional cardiology with the highest doses to patients. Both 
procedures are done by cardiologists with radiological education. By the $\mathrm{CA}$, the cardiologist leads a catheter for application of contrast medium under a fluoroscopy control. After injection of contrast medium to some artery he makes several images by using a pulsed radiography. By the PTCA, the first part of the procedure is the same as by $\mathrm{CA}$, but after the injection of contrast medium the cardiologist provides the treatment of stenoses in patients' arteries. The cardiologist must lead the catheter to the stenosis and dilates this part of the artery using a balloon and he sometimes inserts a stent into the artery to avoid a re-stenosis. This part of the procedure is performed under the fluoroscopy control. After the treatment, the cardiologist makes next images of the coronary artery. Too long fluoro time and great number of cine images are the reasons of the highest doses to patients.

The cardiologist can use plenty of projections of the coronary arteries. But some projections are used very often. If the maximum skin dose is higher than $2 \mathrm{~Gy}$, it can cause a radiation injury. The aim of this study is to determine distribution of $P_{K A}$ values for CA and PTCA, fluoro time for both these procedures, to reconstruct the skin dose distribution for patients undergoing prolonged PTCA procedures, to summarize the most common projections of arteries and to estimate values of $P_{K A}$, fluoro time and number of frames by which the skin dose higher than $2 \mathrm{~Gy}$ is expected.

\section{Material}

For this study, dose data were gained from the General Faculty Hospital in Prague. Information about exposure parameters of patients were recorded by a dose monitoring system of used X-ray machine Siemens AXIOM Artis MP. From the hospital, the dose data for 502 patients undergoing CA and 101 patients undergoing PTCA were available. The information about CA and PTCA comprised total $P_{K A}$, fluoro time, $P_{K A}$ from fluoroscopy, average voltage, average tube current for fluoroscopy and parameters of patients (age, height, weight) as well. Moreover for all PTCA and some CA (73) procedures the dose data comprised further information about exposure parameters such as angle of projection, skin dose for each pulsed radiography sequence, tube voltage, pulse current, pulse width, time of the sequence, thickness of a $\mathrm{Cu}$ filter and focus to image receptor distance. 


\section{Methods}

From the information about angles of projections and skin doses by these projections it was possible to reconstruct skin dose distribution of patients and to determine maximum skin dose. 19 dose distributions from CA and 101 skin dose distributions from the PTCA were reconstructed.

The reconstruction of the dose distribution was performed in MATLAB. The reconstruction in MATLAB is based on adding of the particular fields which are overlapping in the patient surface plane. Total skin dose is computed for each point defined in the plane by summing of doses from each field and then maximum skin dose is determined. By the reconstruction it was presumed that the distribution of projections during fluoroscopy and distribution of fluoroscopy $P_{K A}$ among the fluoroscopy projections is the same as during radiography. A similar presumption was published in study [1]. This presumption was important because no information about angles of projections by the fluoroscopy was available. For determining of the skin dose distribution, matrix of points was defined in the MATLAB. The size of the matrix was 180 columns representing 180 angles between LAO 90 and RAO 90 projections and 120 rows representing 120 angles between CD 60 and CR 60 projections. Distance between the points in the matrix was set to $0.8 \mathrm{~cm}$. Each field in this matrix represented exposed field on the entrance surface plane of a patient. Position of the field in the matrix was determined by intersection of a central beam axis with the patient surface plane. The intersection was computed from angle of projection and source to patient surface plane
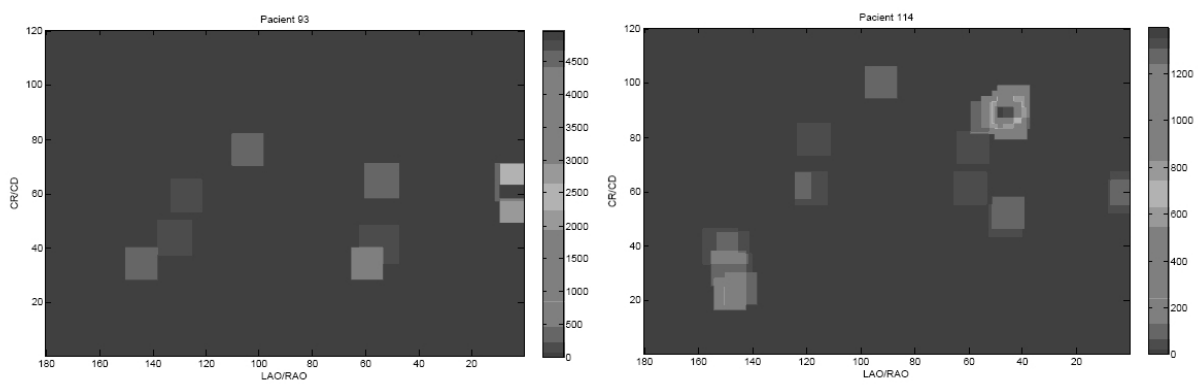

Figure 1. Reconstructed skin dose distributions for two patients undergoing PTCA 
distance, which was taken to be $50 \mathrm{~cm}$. Shift of the intersection in the plane was $0.8 \mathrm{~cm}$ for $1^{\circ}$ change in the angle of projection. This intersection was considered as a centre of square field with dimension 10 to $12 \mathrm{~cm}$ according to source to image distance (SID). To each point of such created field in the matrix, value of skin dose determined by the monitoring system was added. An example of reconstructed dose distributions is shown in Figure 1. The figure represents the view of a back of the patient. The bars beside the graphs indicate the skin dose in mGy.

\section{Results}

Relative contribution of fluoroscopy $P_{K A}$ to total $P_{K A}$ is shown in a distribution in Figure 2 for CA and PTCA.

In Figure 3, the distribution of $P_{K A}$ values for CA and PTCA is shown.

The average values $P_{K A}$, fluoro time and number of frames were determined from the obtained data for comparison with other studies. For CA, the average value (arithmetical mean) of $P_{K A}$ is $50 \mathrm{~Gy} \cdot \mathrm{cm}^{2}$ and the average value of the fluoro time is $5.7 \mathrm{~min}$. For PTCA

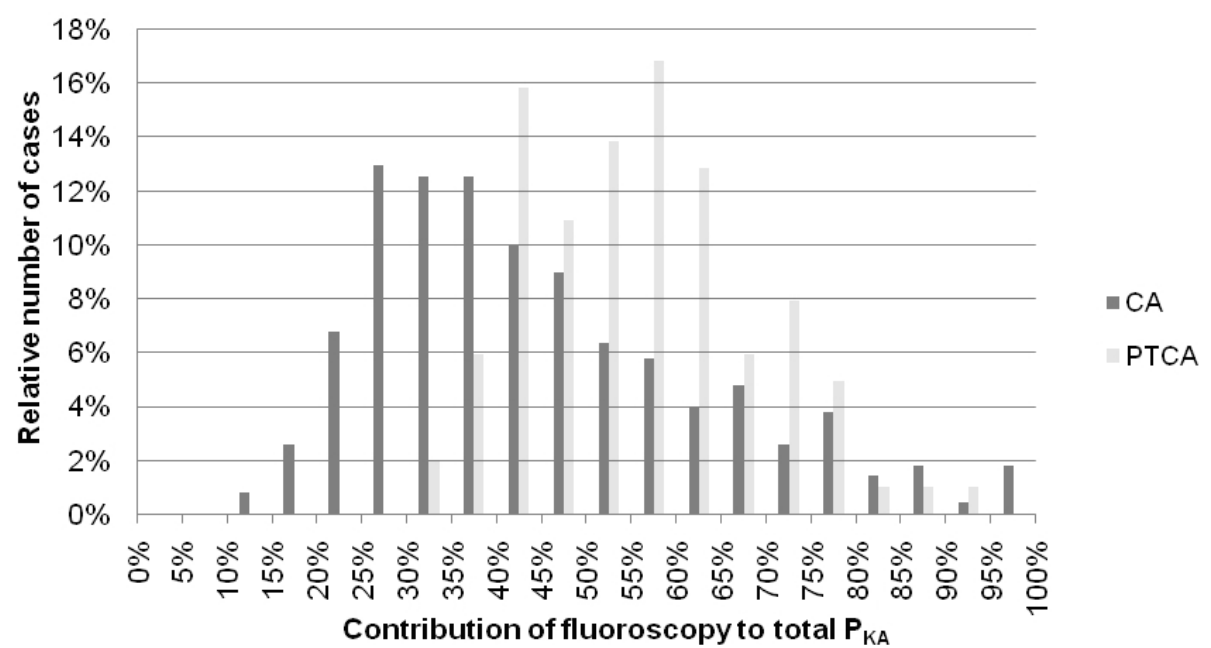

Figure 2. Contribution of fluoroscopy for CA and PTCA 


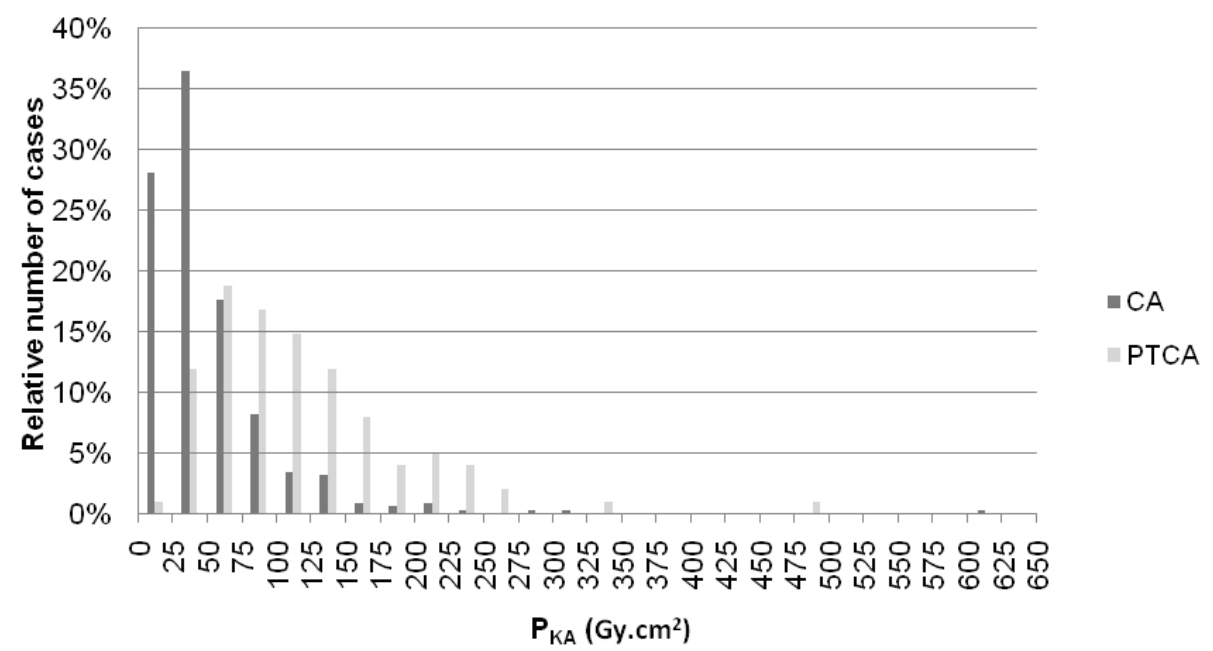

Figure 3. Distribution of $P_{K A}$ for CA and PTCA

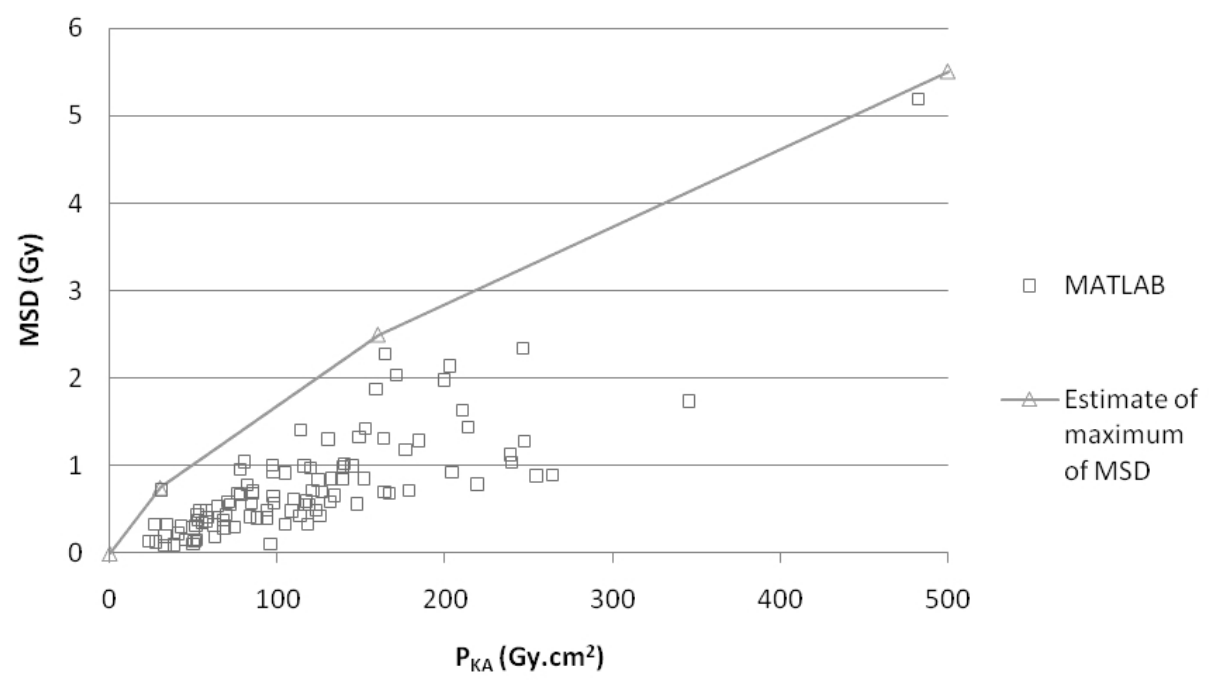

Figure 4. Dependence of maximum skin dose on $P_{K A}$ value 


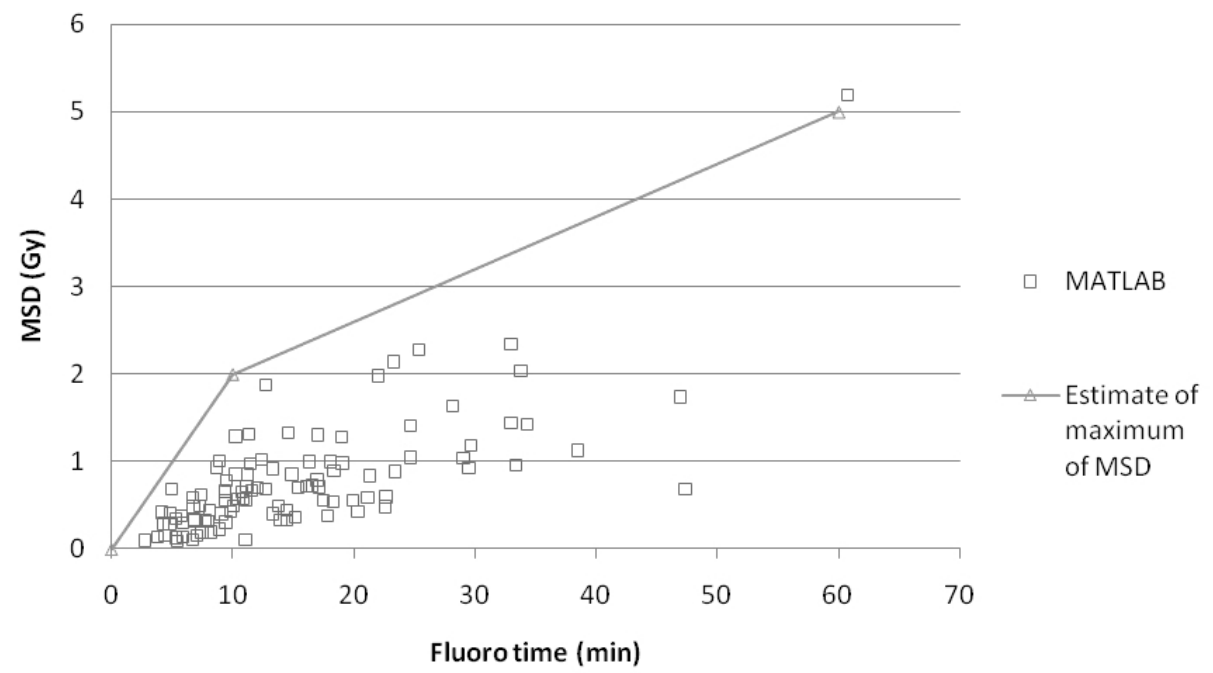

Figure 5. Dependence of maximum skin dose on fluoro time

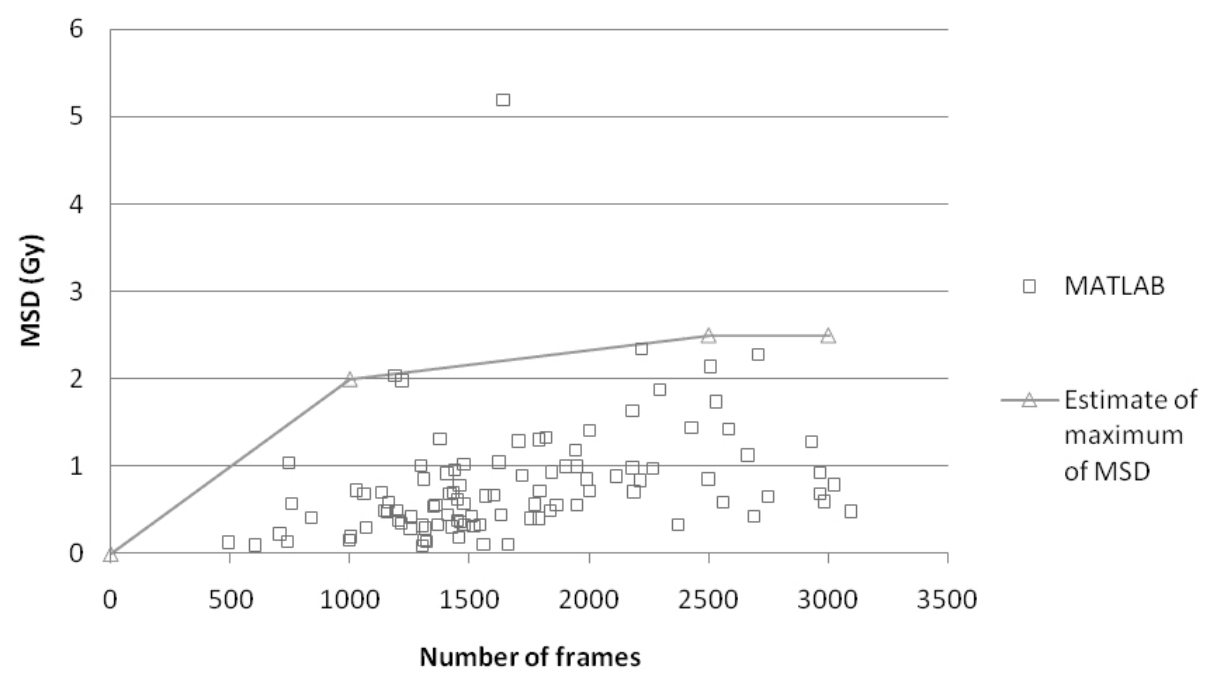

Figure 6. Dependence of maximum skin dose on number of frames 
Table 1. Values of dose related quantities

\begin{tabular}{|c|c|c|c|c|c|c|}
\hline \multirow{2}{*}{ Study } & \multicolumn{3}{|c|}{ CA } & \multicolumn{3}{c||}{ PTCA } \\
\cline { 2 - 7 } & $\begin{array}{c}\text { PAK } \\
{\left[\mathrm{G} y \cdot \mathrm{m}^{2}\right]}\end{array}$ & $\begin{array}{c}\text { Fluoro time } \\
{[\mathrm{min}]}\end{array}$ & $\begin{array}{c}\text { Number of } \\
\text { frames }\end{array}$ & $\begin{array}{c}P_{K A} \\
{\left[\mathrm{G} y \cdot \mathrm{m}^{2}\right]}\end{array}$ & $\begin{array}{c}\text { Fluoro time } \\
{[\mathrm{min}]}\end{array}$ & $\begin{array}{c}\text { Number of } \\
\text { frames }\end{array}$ \\
\hline \hline$[2]$ & 50 & 9 & 1000 & 125 & 22 & 1700 \\
\hline$[3]$ & 57 & 6 & 1270 & 94 & 16 & 1355 \\
\hline$[3]$ & 70 & 5 & 1300 & 120 & 24 & 1800 \\
\hline This study & 50 & 5.7 & - & 118 & 15.4 & 1693 \\
\hline
\end{tabular}

the average values are $118 \mathrm{~Gy} \cdot \mathrm{cm}^{2}$ of $P_{K A}, 15.4$ minutes of fluoro time and 1693 of the number of frames. The comparison with other studies is shown in Table 1.

For CA the parameters of $P_{K A}$ distribution are: the median $36 \mathrm{~Gy} \cdot \mathrm{cm}^{2}$, the third quartile $60 \mathrm{~Gy} \cdot \mathrm{cm}^{2}$. For PTCA the parameters are following: the median $105 \mathrm{~Gy} \cdot \mathrm{cm}^{2}$, the third quartile $149 \mathrm{~Gy} \cdot \mathrm{cm}^{2}$. For CA the parameters of the fluoro time distribution are: the median 3.3 minutes and the third quartile 6.4 minutes. For PTCA: the median 12.3 minutes, the third quartile 19 minutes. The number of frames was determined only for PTCA. The median of the distribution of number of frames is 1543 , the third quartile is 2001 .

From the reconstructed dose distributions the maximum skin dose was determined for each patient. However, it is not possible to perform the reconstruction for each patient, therefore it is advantageous to determine some trigger levels of $P_{K A}$, fluoro time and number of frames which can indicate possible exceeding of the 2 Gy skin dose threshold. Dependence of determined maximum skin doses on the $P_{K A}$, fluoro time and number of frames is shown in Figures 4-6.

From Figure 4 it is evident that for the patients undergoing PTCA with $P_{K A}$ lower than $300 \mathrm{~Gy} \cdot \mathrm{cm}^{2}$ the skin dose higher than $2.5 \mathrm{~Gy}$ is not probable. But for $P_{K A}$ higher than $300 \mathrm{~Gy} \cdot \mathrm{cm}^{2}$, an investigation of the maximum skin doses should be performed for more patients. With regard to the fluoro time and the number of frames it is possible to say that for the fluoro time lower than 35 minutes and the number of frames lower than 3000 it is not expected that the maximum skin dose would exceed 2.5 Gy. 


\section{Discussion}

From Figure 1 it is obvious that by PTCA cardiologists use more fluoroscopy than pulsed radiography. This result was expected because the PTCA is therapeutic procedure, whilst $\mathrm{CA}$ is only diagnostic procedure. The dose monitoring system does not provide information about projections of the fluoroscopy. This is disadvantage of the usually used angiographic systems, because by the reconstruction of the skin dose distribution it has to be supposed, that the projections by the fluoroscopy are the same as by the pulsed radiography. But by a procedure, when the fluoroscopy contributes by 80 to the total $P_{K A}$, this assumption can cause a serious error in the reconstructed dose distribution.

Other disadvantage of the angiographic system is that the system counts with an attenuation of X-ray beam by a patient table. The attenuation of the table is about $20 \%$. By right oblique or left oblique projections the consideration of the attenuation by the table is right, but for lateral projections it causes an underestimation of the skin dose.

A further inaccuracy of the skin dose determination is caused by an indication of skin doses in a reference point, which is $60 \mathrm{~cm}$ far from the focus. The system does not consider a real focus to surface of patient distance.

For improvement of maximum skin dose determination it is necessary to perform a measurement of skin dose of patients by using TLDs or gafchromic films. However, these methods are not used in the Czech Republic and it is not expected that the direct patient dosimetry will be required and established. Therefore the report of the dose monitoring system is the only one information which can be used for the estimate of the maximum skin dose.

\section{Conclusions}

Direct skin dosimetry of each patient undergoing cardiological interventional procedure is not performed in the Czech Republic. Therefore the skin dose distribution and location and value of maximum skin doses are not known. Reconstruction of the dose distribution from the dose monitoring system for each patient is time consuming and therefore not realistic. Therefore, trigger values of kerma-area product, fluoro time and number of frames were determined, which can indicate exceeding of 2 Gy skin dose 
threshold. However, these levels should be verified by analysis of more patient data and by direct patient dosimetry using TLDs or gafchromic films.

From the dose data, most critical projections for each type of PTCA were identified, but the number of patients for each type of PTCA was very small to consider the results statistically significant. To reconstruct the skin dose distribution for more patients of each type of the PTCA is therefore necessary.

A contribution of fluoroscopy and pulsed radiography to total $P_{K A}$ was determined. The distribution of $P_{K A}$ values, fluoro time, skin dose and the distribution of number of frames for PTCA were determined as well. The average values of $P_{K A}$, fluoro time and number of frames are comparable with values published in other studies [2-4].

\section{Acknowledgements}

This study is carried out within a research project of State Office of Nuclear Safety No. 4/2008 'Radiation exposures of patients in interventional radiology' and within a project No. MSM6840770040 'Use of Radionuclides and Ionizing Radiation'.

\section{References}

[1] Bogaert E, Bacher K, Thierens H. A large-scale multicentre study in Belgium of dose area product values and effective doses in interventional cardiology using contemporary X-ray equipment. Rad Prot Dos. 2008; 128: 312-323.

[2] Balter S, Miller DL, Vano E, Ortiz Lopez P, Bernardi G, Cotelo E, Faulkner K, Nowotny R, Padovani R, Ramirez A. A pilot study exploring the possibility of establishing guidance levels in X-ray directed interventional procedures.

Med Phys. 2008; 35(2): 673-680.

[3] Neofotistou V, Vano E, Padovani R, Kotre J, Dowling A, Toivonen M, Kottou S, Tsapaki V, Willis S, Bernardi G, Faulkner K. Preliminary reference levels in interventional cardiology. Eur Radiol. 2003; 13: 2259-2263.

[4] Padovani R, Novario R, Bernardi G. Optimisation in coronary angiography and percutaneous transluminal coronary angioplasty.

Rad Prot Dos. 1998; 80(1-3): 303-306. 